\title{
Stent com Liberação de Everolimus vs. Stent com Liberação de Zotarolimus na Prática Clínica do Mundo Real
}

\author{
Carlos Adolfo Collet ${ }^{1}$, J. Ribamar Costa Jr. ${ }^{1}$, Fausto Feres' ${ }^{1}$, Gustavo Gama ${ }^{1}$, Ricardo Costa', \\ Alejandro Sanchez ${ }^{1}$, Dimytri Siqueira ${ }^{1}$, Daniel Chamié1, Tarcisio Borghi', Rodolfo Staico', \\ Luis Fernando Tanajura ${ }^{1}$, Amanda G. M. R. Sousa ${ }^{1}$, Alexandre Abizaid ${ }^{1}$, J. Eduardo Sousa ${ }^{1}$
}

\section{RESUMO}

Introdução: Os stents com liberação de fármacos antiproliferativos (SF) têm demonstrado sua eficácia nos mais variados cenários. Entretanto, preocupações recentes com a maior ocorrência de trombose muito tardia com a primeira geração de SF, possivelmente associada a reendotelização retardada ou incompleta, impulsionaram o desenvolvimento de dispositivos mais eficazes e seguros. Método: Estudo retrospectivo, de centro único, incluindo pacientes submetidos a intervenção coronária percutânea entre janeiro de 2006 e dezembro de 2008, tratados com SF com liberação de everolimus (everolimus-eluting stent - EES) (Xience $V^{\mathrm{TM}} /$ Promus $^{\mathrm{TM}}$ ) e com SF com liberação de zotarolimus (zotarolimus-eluting stent - ZES) (Endeavor ${ }^{\mathrm{TM}}$ ). O objetivo principal foi comparar a taxa de eventos cardíacos adversos maiores no seguimento de 12 meses entre os pacientes dos grupos EES e ZES. Resultados: No total, 198 pacientes com 266 lesões foram incluídos nesta análise. A média de idade foi de $60 \pm 10$ anos e $39 \%$ tinham diabetes melito, sem diferenças entre os grupos. $\mathrm{O}$ diâmetro de referência $(2,3+0,5 \mathrm{~mm}$ vs. $2,38+0,2 \mathrm{~mm} ; \mathrm{P}=0,14)$ e a extensão da lesão $(16,3 \pm 9,4 \mathrm{~mm}$ vs. $16,1 \pm 11,3 \mathrm{~mm}$; P $=0,89)$ também não diferiram entre os grupos. Após 12 meses de seguimento, a taxa de eventos cardíacos adversos maiores foi de $8,16 \%$ no grupo EES e de $8 \%$ no grupo ZES $(P=0,96)$, sem diferença entre os grupos, com taxa global de revascularização da lesão-alvo de $2 \%$ (1\% EES vs. $3 \%$ $Z E S ; P=0,32$ ). Não houve nenhum caso de trombose definitiva de stent. Conclusão: Nesta análise em pacientes nãoselecionados, as taxas de eventos cardíacos adversos maiores foram semelhantes entre os pacientes dos grupos EES e ZES, com baixas taxas de revascularização da lesão-alvo e excelente perfil de segurança no seguimento de 12 meses.

DESCRITORES: Stents farmacológicos. Restenose coronária. Revascularização miocárdica. Angioplastia transluminal percutânea coronária

\section{ABSTRACT}

Everolimus-Eluting Stent versus Zotarolimus-Eluting Stent in the Real Word Clinical Practice

Background: The use of drug-eluting stents (DES) has proven to be effective in different angiographic scenarios. However, recent concerns with the occurrence of very late stent thrombosis with the first DES generation, probably related to delayed or incomplete endothelization, have led to the development of new safer and more effective devices. Method: Retrospective, single center study, including patients undergoing percutaneous coronary intervention between January 2006 and December 2008, treated with everolimuseluting stent (EES) (Xience $\mathrm{V}^{\mathrm{TM}} /$ Promus $^{\mathrm{TM}}$ ) and zotarolimuseluting stent (ZES) (Endeavor $^{\mathrm{TM}}$ ). Our objective was to compare the rates of major adverse cardiac events between EES and ZES patients after 12 months of follow-up. Results: Overall, 198 patients with 266 lesions were included in the study. Mean age was $60 \pm 10$ years and $39 \%$ had diabetes mellitus, without significant differences between groups. The reference diameter $(2.3 \pm 0.5 \mathrm{~mm}$ vs. $2.38 \pm 0.2 \mathrm{~mm}$; $\mathrm{P}=0.14)$, as well as lesion length $(16.3+9.4 \mathrm{~mm}$ vs. $16.1+$ $11.3 \mathrm{~mm} ; \mathrm{P}=0.89$ ), were also not significantly different between groups. After 12 months of follow-up, the rate of major adverse cardiac events was $8.16 \%$ in the EES group vs. $8 \%$ in the ZES group $(P=0.96)$, with a global rate of target-lesion revascularization of $2 \%$ (1\% EES and $3 \%$ ZES; $P=0.32$ ). There were no cases of definite stent thrombosis. Conclusion: In this study of non-selected patients, the rates of major adverse cardiac events were similar between patients treated in the EES and ZES groups, with low target-lesion revascularization rates and excellent safety profile after 12 months of follow-up.

KEY-WORDS: Drug-eluting stents. Coronary restenosis. Myocardial revascularization. Angioplasty, transluminal, percutaneous coronary.

\footnotetext{
1 Instituto Dante Pazzanese de Cardiologia - São Paulo, SP, Brasil. Correspondência: J. Ribamar Costa Jr. Av. Dr. Dante Pazzanese, 500 - Ibirapuera - São Paulo, SP, Brasil - CEP 04012-180 E-mail: rmvcosta@uol.com.br

Recebido em: 19/9/2010 • Aceito em: 22/11/2010
} 
Collet CA, et al. Stent com Liberação de Everolimus vs. Stent com Liberação de Zotarolimus na Prática Clínica do Mundo Real. Rev Bras Cardiol Invasiva. 2010;18(4):400-6.

A reestenose intrastent, principal limitação dos stents não-farmacológicos, foi significantemente minimizada com o advento dos stents farmacológicos, que demonstraram sua superioridade a curto e longo prazos, diminuindo a necessidade de reintervenção na lesão tratada. ${ }^{1-3}$

Entretanto, preocupações recentes no seguimento tardio dos pacientes tratados com stents farmacológicos de primeira geração (Cypher ${ }^{\mathrm{TM}}$ e Taxus ${ }^{\mathrm{TM}}$ ), ocasionadas pela maior ocorrência de trombose das endopróteses após o primeiro ano, possivelmente secundária a reendotelização retardada ou incompleta e malaposição das hastes do stent na parede arterial, associada a processo inflamatório e remodelamento tardio, impulsionaram o desenvolvimento de dispositivos mais seguros. ${ }^{4-7}$

A segunda geração de stents farmacológicos, desenhada com plataformas de baixo perfil com hastes mais finas, polímeros mais biocompatíves ou biodegradáveis, e drogas mais eficazes (Figura 1), demonstrou sua efetividade quando comparada com stents farmacológicos de primeira geração. ${ }^{8,9}$ Entre os denominados stents farmacológicos de segunda geração, dois se destacam pelo programa clínico mais desenvolvido: o stent farmacológico com liberação de zotarolimus (zotarolimus-eluting stent - ZES) (Endeavor ${ }^{\mathrm{TM}}$ ) e o stent farmacológico com liberação de everolimus (everolimuseluting stent - EES) (Xience $\mathrm{V}^{\mathrm{TM}} /$ Promus $^{\mathrm{TM}}$ ).
Embora esses dois novos stents farmacológicos tenham sido comparados aos stents não-farmacológi$\cos ^{10,11}$ e aos stents farmacológicos de primeira geração ${ }^{8,9}$, com resultados encorajadores, sobretudo a longo prazo, são raras as comparações entre os stents farmacológicos de segunda geração, especialmente fora do cenário dos estudos controlados.

No presente estudo objetivamos comparar os desfechos clínicos em um ano de uma população nãoselecionada tratada com stent farmacológico com liberação de everolimus (Xience $V^{\mathrm{TM}} /$ Promus $^{\mathrm{TM}}$ ) e com stent farmacológico com liberação de zotarolimus (Endeavor ${ }^{\mathrm{TM}}$ ).

\section{MÉTODO}

\section{Desenho do estudo}

Estudo retrospectivo, de centro único, comparando pacientes submetidos a intervenção coronária percutânea entre janeiro de 2006 e dezembro de 2008 tratados com stent farmacológico com liberação de everolimus (Xience $\mathrm{V}^{\mathrm{TM}} /$ Promus $^{\mathrm{TM}}$ ) (grupo EES) e tratados com stent farmacológico com liberação de zotarolimus (Endeavor ${ }^{\mathrm{TM}}$ ) (grupo ZES).

Foram poucos os critérios de exclusão deste estudo: pacientes tratados na vigência de infarto agudo do miocárdio, lesões em ponte de veia safena e lesões em bifurcações coronárias necessitando de dois ou mais stents.

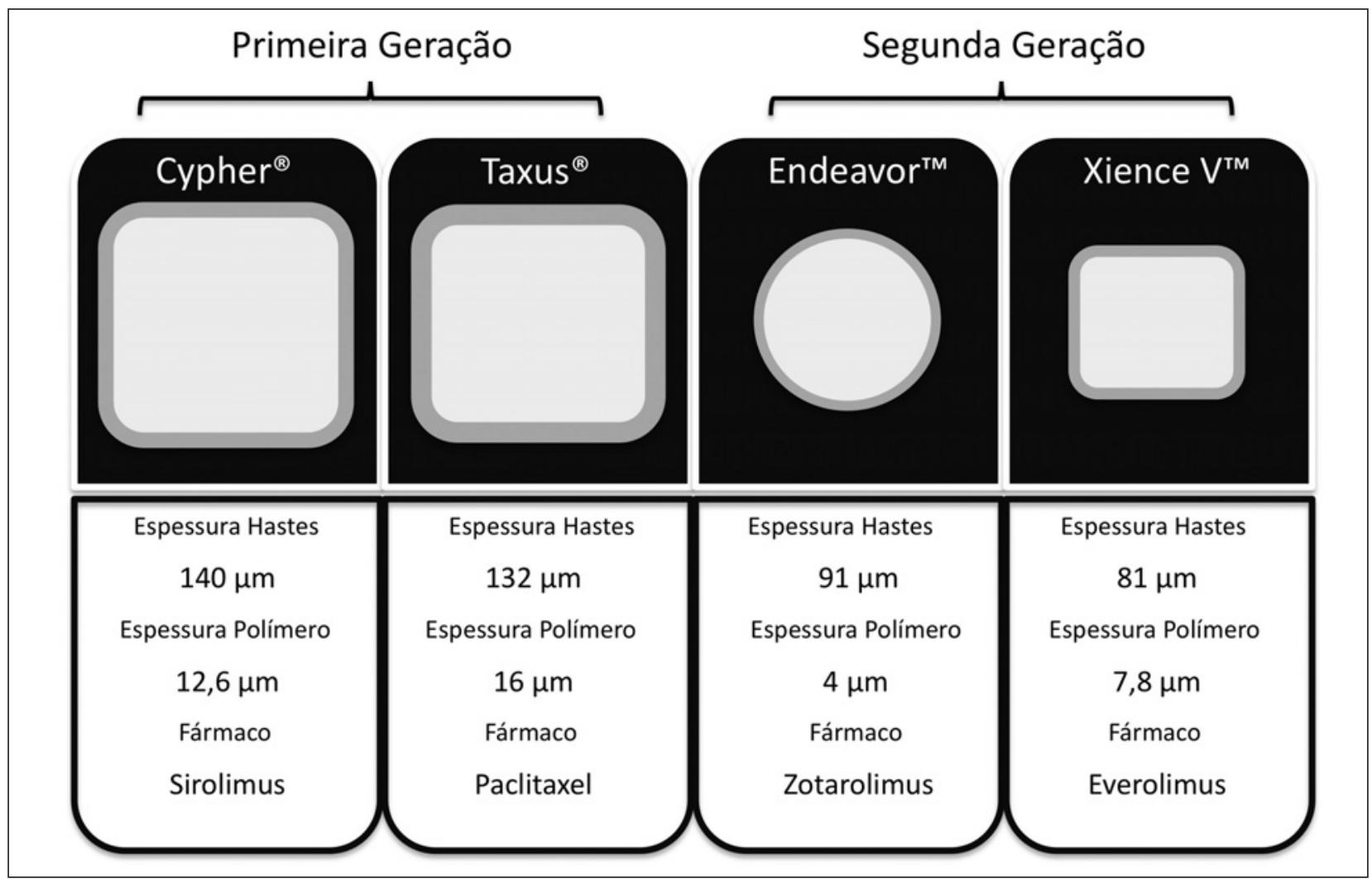

Figura 1 - Espessura das hastes e dos polímeros dos stents farmacológicos de primeira e de segunda geração. (Adaptado de Maluenda et al. ${ }^{8}$ ) 
O acompanhamento clínico foi realizado por consulta médica ou por contato telefônico. Declaração de consentimento informado foi obtida de todos os pacientes e o protocolo foi aprovado pelo Comitê de Ética de nossa instituição.

\section{Descrição dos dispositivos}

\section{1) Stent com liberação de everolimus}

O stent Xience $\mathrm{V}^{\mathrm{TM}} /$ Promus $^{\mathrm{TM}}$ tem plataforma flexível de cobalto-cromo no sistema de entrega ML VISION ${ }^{\mathrm{TM}}$ com hastes finas $(81 \mu \mathrm{m})$, cobertas por um polímero biocompatível durável fluorado de 7,8 $\mu \mathrm{m}$ de espessura, composto pelos monômeros VDF e HFP, que permitem mais elasticidade, minimizando possíveis dilacerações do polímero durante a expansão do stent. O everolimus, um macrolídeo semissintético, imunossupressor, é um análogo da rapamicina, mais potente, que, a sua semelhança, se une ao FKBP12 citosólico, bloqueando o ciclo celular entre as fases G1 e S e inibindo a proliferação das células musculares lisas. ${ }^{12}$

\section{2) Stent com liberação de zotarolimus}

O stent Endeavor ${ }^{\mathrm{TM}}$ tem plataforma de estrutura modular de cromo-cobalto, com hastes de $91 \mu \mathrm{m}$ de espessura, que confere flexibilidade e facilidade na entrega do dispositivo. O polímero biocompatível, fosforilcolina, de $4 \mu \mathrm{m}$ de espessura, assemelha-se à camada externa dos glóbulos vermelhos. O zotarolimus é um fármaco no qual o anel tetrazol na molécula de sirolimus foi substituído por um grupo hidroxil $(\mathrm{OH})$, resultando em maior lipofilidade e penetração na parede arterial, com propriedades antiproliferativas e anti-inflamatórias. Em estudos pré-clínicos, aproximadamente 50\% do zotarolimus é liberado nos primeiros dias após o implante, no entanto pode-se detectar o fármaco no tecido perivascular até 30 dias do procedimento. ${ }^{13}$

\section{Procedimento e intervenção coronária}

Todos os procedimentos foram realizados de acordo com as diretrizes vigentes. ${ }^{14} \mathrm{O}$ protocolo antiplaquetário envolveu a utilização de ácido acetilsalicílico (dose de ataque, 300 mg; dose de manutenção, 100 mg/ dia) e de clopidogrel (dose de ataque, 300-600 mg; dose de manutenção, $75 \mathrm{mg} /$ dia), iniciada 24 horas antes do procedimento. Após intervenção, o ácido acetilsalićlico foi mantido indefinidamente e o clopidogrel foi mantido por 3 meses nos casos de implante de stent com liberação de zotarolimus e por 12 meses nos casos de stent com liberação de everolimus. Durante o procedimento, heparina não-fracionada foi administrada para todos os pacientes na dose de $100 \mathrm{UI} / \mathrm{kg}$, com o objetivo de manter o tempo de coagulação ativada entre 250-300 segundos. O uso dos inibidores da glicoproteína Ilb/Illa ficou a critério dos operadores, assim como a realização de pré e pós-dilatação.

\section{OBJETIVOS E DEFINIÇÕES}

O objetivo primário foi comparar as taxas de eventos clínicos adversos maiores, definidos como ocorrência de morte cardíaca, infarto do miocárdio e necessidade de revascularização da lesão-alvo dentro do primeiro ano após o procedimento.

Como objetivo secundário buscou-se identificar os preditores da falência do tratamento percutâneo, definida como revascularização da lesão-alvo ou oclusão total intrastent confirmada angiograficamente.

Todos os óbitos foram considerados de origem cardíaca, a menos que uma causa não-cardíaca fosse inequivocamente identificada. Infarto periprocedimento foi definido como elevação dos níveis de creatina quinase fração $\mathrm{MB}(\mathrm{CK}-\mathrm{MB})$ maior que três vezes o valor normal. A revascularização da lesão-alvo foi definida como novo procedimento percutâneo ou cirúrgico decorrente de reestenose do segmento da lesão-alvo. Todas as revascularizações da lesão-alvo foram guiadas por sintomas ou por evidência objetiva de isquemia em testes não-invasivos. Trombose de stent foi definida segundo os critérios do Academic Research Consortium (ARC), sendo classificada como definitiva, provável ou possível, e como aguda (até 24 horas), subaguda (24 horas até 30 dias) e tardia (entre 30 dias e um ano). ${ }^{15}$ Como o presente estudo avalia o seguimento clínico até um ano, as taxas de trombose muita tardia (> um ano) não serão apresentadas.

Sucesso angiográfico foi definido como fluxo TIMI 3 ao final do procedimento com lesão residual $<30 \%$ e sucesso clínico, como sucesso angiográfico sem eventos clínicos adversos maiores no seguimento intrahospitalar. Nos casos de lesões em bifurcações coronárias o tratamento do ramo lateral foi realizado apenas com cateter-balão, com critério angiográfico de sucesso de lesão residual $<50 \%$, fluxo TIMI 3 e ausência de dissecções.

\section{Análise estatística}

As variáveis quantitativas são apresentadas como média \pm desvio padrão e as qualitativas, como números absolutos e porcentagens. Para a comparação dos grupos usou-se o teste $t$ de Student e o teste exato de Fisher. Usou-se o método de Kaplan-Meier para a apresentação das curvas de sobrevida livre de eventos e compararam-se os grupos com o log-rank test. O valor de $\mathrm{P} \leq 0,05$ foi considerado significativo. $\mathrm{O}$ modelo de regressão logística de Cox foi utilizado para identificar os preditores de falência do tratamento percutâneo. A análise estatística foi realizada com o software SPSS versão 16.0 (SPSS Inc., Chicago, Estados Unidos).

\section{RESULTADOS}

No total, foram incluídos nesta análise 198 pacientes com 266 lesões (128 no grupo EES e 138 no grupo 
Collet CA, et al. Stent com Liberação de Everolimus vs. Stent com Liberação de Zotarolimus na Prática Clínica do Mundo Real. Rev Bras Cardiol Invasiva. 2010;18(4):400-6.

ZES). A média de idade foi de $60 \pm 10$ anos, sendo $45 \%$ do sexo feminino e $39 \%$ portadores de diabetes melito, sem diferenças significativas entre os grupos. Em relação à indicação clínica, no grupo EES a síndrome coronária aguda foi significativamente mais frequente que no grupo ZES (26,5\% vs. $13 \%$, respectivamente; $P=0,01)$. Outras características clínicas são apresentadas na Tabela 1.

As características angiográficas e do procedimento são apresentadas na Tabela 2. O vaso mais frequentemente tratado no grupo EES foi a artéria descendente anterior, enquanto no grupo ZES predominou a coronária direita $(47 \%$ vs. $39 \% ; P=0,06)$. Mais de $75 \%$ das lesões eram do tipo B2/C (classificação do American College of Cardiology/American Heart Association), sem diferenças entre os grupos. Houve significativamente mais pré e pós-dilatação no grupo EES que no grupo ZES $(65,7 \%$ vs. $47,5 \%$, $\mathrm{P}<0,01$, e $85,8 \%$ vs. $36,6 \%$, $\mathrm{P}<0,01$, respectivamente). Também houve maior porcentagem de lesões em bifurcações no grupo EES comparativamente ao grupo ZES (16\% vs. 1\%, respectivamente; $\mathrm{P}<0,01)$. Em relação à análise angiográfica quantitativa, não houve diferenças quanto a extensão da lesão, diâmetro de referência e estenose residual entre os grupos.

Ao longo de 12 meses de seguimento houve 16 eventos adversos, igualmente distribuídos entre os dois grupos (Tabela 3). Na fase intra-hospitalar, 5 pacientes de cada grupo apresentaram infarto periprocedimento. Ao final de 12 meses de seguimento, a taxa de eventos clínicos adversos maiores foi de $8,16 \%$ no grupo EES e de $8 \%$ no grupo ZES $(P=0,96)$, sem diferença significativa entre os grupos (Figura 2), com taxa global de revascularização da lesão-alvo de $2 \%$ (1\% no grupo EES e $3 \%$ no grupo ZES; $P=0,32$ ). Em relação à trombose de stent, houve uma morte súbita 20 dias após o tratamento percutâneo, classificado como trombose provável, no grupo EES.
Em relação à falência do tratamento percutâneo, houve 4 revascularizações da lesão-alvo e 2 casos de reestudo invasivo guiado por sintomas, nos quais o achado foi oclusão total do vaso com circulação colateral (1 no grupo EES e 1 no grupo ZES), cujo manejo foi apenas com tratamento clínico. $\mathrm{Na}$ análise univariada, identificaram-se como preditores de falência do tratamento percutâneo implante de 2 ou mais stents [odds ratio (OR) 8,2, intervalo de confiança de 95\% (IC 95\%) 1,4-46,7; P = 0,05), extensão total maior de 30 mm (OR 4,21, IC 95\% 0,98-19,34; P = 0,046) e diâmetro do vaso $\leq 2,5 \mathrm{~mm}$ (OR 12, IC 95\% 1,4$101,2 ; P=0,04)$. Após análise multivariada, o diâmetro do vaso $\leq 2,5 \mathrm{~mm}$ foi o único preditor independente de falência do tratamento percutâneo (OR 13,1, IC 95\% 1,5-113; $P=0,01)$.

\section{DISCUSSÃO}

O principal achado deste estudo é a equivalência, dentro do primeiro ano de acompanhamento, dos desfechos clínicos de pacientes minimamente selecionados, tratados com stents com liberação de everolimus e de zotarolimus.

Após os primeiros estudos em pacientes selecionados, o stent com liberação de everolimus foi avaliado no cenário da prática clínica do mundo real no estudo COMPARE, em que 1.800 pacientes, com mínimos critérios de exclusão, foram randomizados para receber stent com liberação de everolimus ou stent com liberação de paclitaxel. Ao final de 12 meses, o desfecho primário (morte, infarto do miocárdio e revascularização do vaso-alvo) foi significativamente menor no grupo tratado com stent com liberação de everolimus em comparação com o grupo que recebeu stent com liberação de paclitaxel $(6 \%$ vs. $9 \%$, respectivamente; $P=0,02)$. Essa diferença decorreu de uma taxa 10 vezes menor de trombose (definitiva/provável) e infarto do miocárdio com o stent com liberação de everolimus

TABELA 1

Características clínicas basais

\begin{tabular}{lccc} 
Variável & EES $(\mathbf{n}=\mathbf{9 8})$ & ZES $(\mathbf{n}=\mathbf{1 0 0})$ & Valor de P \\
\hline Idade, anos & $59,8 \pm 1$ & $60,8 \pm 1$ & 0,53 \\
Sexo masculino, n (\%) & $43(44)$ & $67(67)$ & 0,01 \\
Hipertensão, n (\%) & $85(86,7)$ & $81(81)$ & 0,27 \\
Dislipidemia, n (\%) & $69(70,4)$ & $63(63)$ & 0,26 \\
Diabetes melito, n (\%) & $44(45)$ & $34(34)$ & 0,11 \\
Tabagismo, n (\%) & $16(16,3)$ & $11(11)$ & 0,27 \\
IAM prévio, n (\%) & $45(47,4)$ & $50(50)$ & 0,56 \\
IRC, n (\%) & $5(5,1)$ & $4(4)$ & 0,89 \\
SCA, n (\%) & $26(26,5)$ & $13(13)$ & 0,01 \\
\hline
\end{tabular}

$\mathrm{EES}=$ stent com liberação de everolimus (everolimus-eluting stent); IAM = infarto agudo do miocárdio; IRC = insuficiência renal crônica; $\mathrm{n}=$ número de pacientes; $\mathrm{SCA}$ = síndrome coronária aguda; ZES = stent com liberação de zotarolimus (zotarolimus-eluting stent). 
comparativamente ao stent com liberação de paclitaxel $(0,2 \%$ vs. $2 \%, P=0,002$ e $3 \%$ vs. $5 \%, P=0.007$, respectivamente) ${ }^{16}$, demonstrando superioridade do stent Xience $V^{T M}$ em relação ao stent de primeira geração Taxus ${ }^{T M}$.

Do mesmo modo, o stent com liberação de zotarolimus Endeavor ${ }^{\mathrm{TM}}$ foi comparado, no cenário do mundo real, com o stent com liberação de sirolimus (sirolimus-eluting stent - SES) no estudo SORT OUT III, que incluiu 2.332 pacientes assim distribuídos: 1.162 pacientes no grupo que recebeu stent com liberação de zotarolimus (grupo ZES) e 1.170 no grupo que recebeu stent com liberação de sirolimus (grupo SES). Após 18 meses de seguimento clínico, o grupo tratado com SES foi superior quanto ao desfecho primário combinado de morte cardíaca, infarto do miocárdio e revascularização do vaso-alvo $(10 \%$ no grupo ZES vs. $5 \%$ no grupo SES; $\mathrm{P}<0,0001)$, com menor taxa de

TABELA 2

Características angiográficas e do procedimento

\begin{tabular}{|c|c|c|c|}
\hline Variável & EES (98 pacientes) & ZES (100 pacientes) & Valor de $\mathbf{P}$ \\
\hline Lesões, n & 128 & 138 & \\
\hline \multicolumn{4}{|l|}{ Vaso tratado } \\
\hline $\mathrm{DA} / \mathrm{Dg}, \mathrm{n}(\%)$ & $64(47)$ & $50(36)$ & 0,06 \\
\hline $\mathrm{CX} / \mathrm{Mg}, \mathrm{n}(\%)$ & $39(28,7)$ & $34(24)$ & 0,42 \\
\hline$C D, n(\%)$ & $32(23,5)$ & $54(39)$ & $<0,01$ \\
\hline Pré-dilatacão, n (\%) & $88(65,7)$ & $66(47,5)$ & $<0,01$ \\
\hline Pós-dilatação, n (\%) & $115(85,8)$ & $51(36,6)$ & $<0,01$ \\
\hline Stent, $\mathrm{n}$ & 182 & 171 & - \\
\hline Stent por paciente, $\mathrm{n}$ & $1,85 \pm 0,73$ & $1,71 \pm 0,98$ & 0,25 \\
\hline Extensão stent por lesão, mm & $27,6 \pm 13,9$ & $23,0 \pm 13,9$ & 0,38 \\
\hline Bifurcação, n (\%) & $21(16)$ & $1(1)$ & $<0,01$ \\
\hline Inibidor da glicoproteína IIb/IIla, n (\%) & $4(4,1)$ & $2(2)$ & 0,44 \\
\hline \multicolumn{4}{|l|}{ Classificação AHA/ACC, \% } \\
\hline A & 11,6 & 1,9 & \multirow{4}{*}{0,06} \\
\hline B1 & 9,1 & 17,4 & \\
\hline B2 & 45,8 & 61,4 & \\
\hline $\mathrm{C}$ & 33,5 & 19,3 & \\
\hline \multicolumn{4}{|l|}{ Angiografia coronária quantitativa } \\
\hline Extensão da lesão, mm & $16,3 \pm 9,4$ & $16,1 \pm 11,3$ & 0,89 \\
\hline Diâmetro de referência, mm & $2,3 \pm 0,5$ & $2,38 \pm 0,2$ & 0,14 \\
\hline Diâmetro luminal mínimo, mm & $0,82 \pm 0,41$ & $0,79 \pm 0,23$ & 0,52 \\
\hline Porcentagem de estenose, \% & $64,2 \pm 16,3$ & $67 \pm 14,5$ & 0,2 \\
\hline Estenose residual, \% & $9,1 \pm 3,7$ & $8,64 \pm 0,9$ & 0,23 \\
\hline
\end{tabular}

$\mathrm{CD}$ = artéria coronária direita; $\mathrm{Cx}=$ artéria circunflexa; $\mathrm{DA}=$ artéria descendente anterior; $\mathrm{Dg}$ = ramo diagonal; $\mathrm{EES}=$ stent com liberação de everolimus (everolimus-eluting stent); $\mathrm{Mg}$ = marginal; $\mathrm{n}$ = número; $\mathrm{ZES}$ = stent com liberação de zotarolimus (zotarolimus-eluting stent).

TABELA 3

Eventos adversos em 12 meses

\begin{tabular}{lccc}
\hline Variável & EES $(\mathbf{n}=\mathbf{9 8})$ & ZES $(\mathbf{n}=\mathbf{1 0 0})$ & Valor de P \\
\hline Óbito cardíaco, $\mathrm{n}(\%)$ & $1(1)$ & 0 & 0,49 \\
IAM, $\mathrm{n}(\%)$ & $7(7,1)^{*}$ & $5(5)^{*}$ & 0,52 \\
RLA, $\mathrm{n}(\%)$ & $1(1)$ & $3(3)$ & 0,32 \\
ECAM, $\mathrm{n}(\%)$ & $8(8,16)$ & $8(8)$ & 0,96 \\
\hline
\end{tabular}

* 5 casos de infarto agudo do miocárdio pós-procedimento em cada grupo.

ECAM = eventos cardíacos adversos maiores; $\mathrm{EES}$ = stent com liberação de everolimus (everolimus-eluting stent); IAM = infarto agudo do miocárdio; $\mathrm{n}$ = número de pacientes; RLA = revascularização da lesão-alvo; ZES = stent com liberação de zotarolimus (zotarolimus-eluting stent). 
Collet CA, et al. Stent com Liberação de Everolimus vs. Stent com Liberação de Zotarolimus na Prática Clínica do Mundo Real. Rev Bras Cardiol Invasiva. 2010;18(4):400-6.

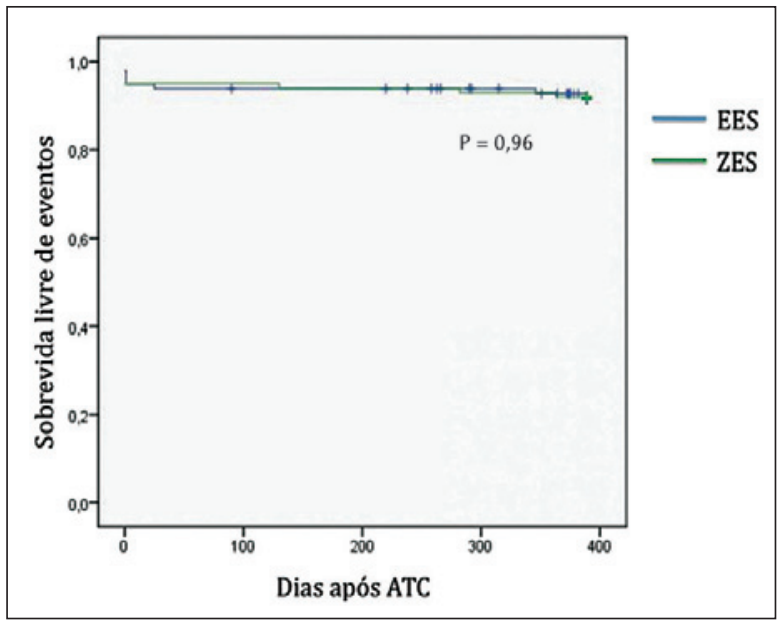

Figura 2 - Curva de Kaplan-Meier para sobrevida livre de eventos cardíacos adversos maiores entre pacientes tratados com stent com liberação de everolimus (EES) e stent com liberação de zotarolimus (ZES). ATC = angioplastia transluminal coronária.

infarto do miocárdio ( $2 \%$ no grupo ZES vs. $1 \%$ no grupo SES; $P=0,029)$ e necessidade de revascularização da lesão-alvo ( $6 \%$ no grupo ZES vs. $2 \%$ no grupo SES; $\mathrm{P}<0,0001)$, mas sem diferença nas taxas de trombose definitiva de stent $(1,1 \%$ no grupo ZES vs. $0,5 \%$ no grupo SES; $\mathrm{P}=0,13) \cdot{ }^{17}$

Na nossa população, os desfechos no primeiro ano de seguimento foram semelhantes entre os grupos EES e ZES, no entanto com taxas muito baixas de revascularização da lesão-alvo em ambos os grupos (1\% no grupo EES vs. 3\% no grupo ZES), especialmente em comparação com outras publicações de pacientes não-selecionados. ${ }^{16-18}$ Essa diferença pode ser atribuída à menor complexidade angiográfica de nossos pacientes, sem lesões em bifurcações que necessitassem dois ou mais stents, oclusões crônicas ou reestenoses intrastent.

\section{Limitações do estudo}

As principais limitações do presente estudo dizem respeito a seu desenho não-randomizado e ao tamanho da população incluída, que pode ser insuficiente para avaliar desfechos clínicos. Ainda, o tempo de terapia antiplaquetária dupla distinto para os dois grupos pode ter tido algum impacto nos desfechos, ainda que não esteja bem claro na literatura a questão do risco/ benefício da terapia dupla prolongada.

\section{CONCLUSÃO}

Nesta análise em pacientes minimamente selecionados, as taxas de eventos adversos cardíacos maiores foram semelhantes entre o stent com liberação de everolimus e o stent com liberação de zotarolimus, com baixas taxas de revascularização da lesão-alvo e trombose do stent no seguimento de 12 meses.

\section{CONFLITO DE INTERESSES}

Os autores declararam inexistência de conflito de interesses relacionado a este manuscrito.

\section{REFERÊNCIAS}

1. Morice MC, Serruys PW, Sousa JE, Fajadet J, Ban Hayashi E, Perin M, et al.; RAVEL Study Group. A randomized comparison of a sirolimus-eluting stent with a standard stent for coronary revascularization. N Engl J Med. 2002;346(23):1773-80.

2. Caixeta A, Leon MB, Lansky AJ, Nikolsky E, Aoki J, Moses JW, et al. 5-year clinical outcomes after sirolimus-eluting stent implantation insights from a patient-level pooled analysis of 4 randomized trials comparing sirolimus-eluting stents with bare-metal stents. J Am Coll Cardiol. 2009;54(10):894-902.

3. Grube E, Dawkins K, Guagliumi G, Banning A, Zmudka K, Colombo A, et al. TAXUS VI final 5-year results: a multicentre, randomised trial comparing polymer-based moderate-release paclitaxel-eluting stent with a bare metal stent for treatment of long, complex coronary artery lesions. Eurolntervention. 2009;4(5):572-7.

4. Holmes DR Jr, Kereiakes DJ, Laskey WK, Colombo A, Ellis SG, Henry TD, et al. Thrombosis and drug-eluting stents: an objective appraisal. J Am Coll Cardiol. 2007;50(2):109-18.

5. Daemen J, Wenaweser P, Tsuchida K, Abrecht L, Vaina S, Morger $\mathrm{C}$, et al. Early and late coronary stent thrombosis of sirolimus-eluting and paclitaxel-eluting stents in routine clinical practice: data from a large two-institutional cohort study. Lancet. 2007;369(9562):667-78.

6. Roukoz H, Bavry AA, Sarkees ML, Mood GR, Kumbhani DJ, Rabbat $M G$, et al. Comprehensive meta-analysis on drugeluting stents versus bare-metal stents during extended followup. Am J Med. 2009;122(6):581.e1-10.

7. Cook S, Ladich E, Nakazawa G, Eshtehardi P, Neidhart M, Vogel R, et al. Correlation of intravascular ultrasound findings with histopathological analysis of thrombus aspirates in patients with very late drug-eluting stent thrombosis. Circulation. 2009;120(5):391-9.

8. Maluenda G, Lemesle G, Waksman R. A critical appraisal of the safety and efficacy of drug-eluting stents. Clin Pharmacol Ther. 2009;85(5):474-80.

9. Leon MB, Mauri L, Popma JJ, Cutlip DE, Nikolsky E, O'Shaughnessy C, et al. A randomized comparison of the ENDEAVOR zotarolimus-eluting stent versus the TAXUS paclitaxel-eluting stent in de novo native coronary lesions 12-month outcomes from the ENDEAVOR IV trial. J Am Coll Cardiol. 2010;55(6):543-54.

10. Stone GW, Rizvi A, Newman W, Mastali K, Wang JC, Caputo R, et al. SPIRIT IV Investigators. Everolimus-eluting versus paclitaxel-eluting stents in coronary artery disease. $\mathrm{N}$ Engl J Med. 2010;362(18):1663-74.

11. Wiemer M, Serruys PW, Miquel-Hebert K, Neumann FJ, Piek JJ, Grube E, et al. Five-year long-term clinical follow-up of the XIENCE $V$ everolimus eluting coronary stent system in the treatment of patients with de novo coronary artery lesions: the SPIRIT FIRST trial. Catheter Cardiovasc Interv. 2010; 75(7):997-1003.

12. Eisenstein EL, Wijns W, Fajadet J, Mauri L, Edwards R, Cowper PA, et al.; ENDEAVOR III Investigators. Long-term clinical and economic analysis of the Endeavor drug-eluting stent versus the Driver bare-metal stent: 4-year results from the ENDEAVOR II trial (Randomized Controlled Trial to Evaluate the Safety and Efficacy of the Medtronic AVE ABT-578 Eluting Driver Coronary Stent in De Novo Native Coronary Artery Lesions). JACC Cardiovasc Interv. 2009;2(12):1178-87.

13. Claessen BE, Caixeta A, Henriques JP, Piek JJ. Current status 
Collet CA, et al. Stent com Liberação de Everolimus vs. Stent com Liberação de Zotarolimus na Prática Clínica do Mundo Real. Rev Bras Cardiol Invasiva. 2010;18(4):400-6.

of the Xience $\mathrm{V}^{\circledR}$ everolimus-eluting coronary stent system. Expert Rev Cardiovasc Ther. 2010;8(10):1363-74.

14. Kandzari DE. Development and performance of the zotarolimus-eluting Endeavor coronary stent. Expert Rev Med Devices. 2010;7(4):449-59.

15. Kushner FG, Hand M, Smith SC Jr, King SB $3^{\text {rd }}$, Anderson JL, Antman EM, et al. 2009 focused updates: ACC/AHA guidelines for the management of patients with ST-elevation myocardial infarction (updating the 2004 guideline and 2007 focused update) and ACC/AHA/SCAI guidelines on percutaneous coronary intervention (updating the 2005 guideline and 2007 focused update): a report of the American College of Cardiology Foundation/American Heart Association Task Force on Practice Guidelines. J Am Coll Cardiol. 2009;54(23):2205-41.
16. Cutlip DE, Windecker S, Mehran R, Boam A, Cohen DJ, van Es GA, et al.; Academic Research Consortium. Clinical end points in coronary stent trials: a case for standardized definitions. Circulation. 2007;115(17):2344-51.

17. Kedhi E, Joesoef KS, McFadden E, Wassing J, van Mieghem C, Goedhart D, et al. Second-generation everolimus-eluting and paclitaxel-eluting stents in real-life practice (COMPARE): a randomised trial. Lancet. 2010;375(9710):201-9.

18. Rasmussen K, Maeng M, Kaltoft A, Thayssen P, Kelbaek H, Tilsted HH, et al.; SORT OUT III Study Group. Efficacy and safety of zotarolimus-eluting and sirolimus-eluting coronary stents in routine clinical care (SORT OUT III): a randomised controlled superiority trial. Lancet. 2010;375(9720): 1090-9. 